\title{
Additions to the lists of publications of Terry Erwin, taxa described by him and named after him after 2015
}

\author{
Lyubomir Penev ${ }^{1,2}$, Achille Casale², Yordanka Banalieva² \\ I Institute of Biodiversity \& Ecosystem Research - Bulgarian Academy of Sciences, Sofia, Bulgaria 2 Pensoft Pub- \\ lishers, Sofia, Bulgaria 3 University of Sassari, Italy (Zoology). Private: Corso Raffaello 12, 10126 Torino, Italy \\ Corresponding author: Lyubomir Penev (1.penev@pensoft.net)
}

Academic editor: James K. Liebherr | Received 1 June 2021 | Accepted 8 June 2021 | Published 16 June 2021

http://zoobank.org/59DC10CC-261D-4E03-9D04-58151C8A2C67

Citation: Penev L, Casale A, Banalieva Y (2021) Additions to the lists of publications of Terry Erwin, taxa described by him and named after him after 2015. In: Spence J, Casale A, Assmann T, Liebherr JK, Penev L (Eds) Systematic Zoology and Biodiversity Science: A tribute to Terry Erwin (1940-2020). ZooKeys 1044: 1037-1042. https://doi.org/10.3897/ zookeys.1044.69603

\begin{abstract}
A paper on the occasion of the $75^{\text {th }}$ birthday of Terry Lee Erwin (1940-2020), an outstanding biologist and founding Editor-in-Chief of ZooKeys, was published in 2015 and contained complete lists of Erwin's publications, patronyms (taxa named after him) and new taxa published by him. The present paper aims to complement these lists with all new information published after 2015, including the papers in the present special issue of ZooKeys dedicated to the blessed memory of Terry Lee Erwin.
\end{abstract}

\section{Keywords}

Biodiversity, bibliography, Carabidae, Coleoptera, Hemiptera, Hymenoptera, Insecta, patronyms

\section{Introduction}

The ZooKeys founding Editor-in-Chief and world-known entomologist Terry Lee Erwin passed away on $11^{\text {th }}$ of May 2020, leaving a sad feeling of emptiness and loss in our hearts! In recognition of his outstanding scientific achievements and dedication to ZooKeys from the very start of the journal, Pensoft and Terry's colleagues and friends organised a memorial volume in ZooKeys published today and of which the present article is a part. It encompasses the list of 10 publications of Terry Erwin, 31 new taxa described by him and 16 taxa named after him, published after the Festschrift paper on the occasion of Terry's $75^{\text {th }}$ birthday (ZooKeys Editorial Office 2015), including also

Copyright Lyubomir Penev et al. This is an open access article distributed under the terms of the Creative Commons Attribution License (CC BY 4.0), which permits unrestricted use, distribution, and reproduction in any medium, provided the original author and source are credited. 
the patronyms described to honor Terry in the present memorial volume. In total, this makes 268 publications, 67 patronyms and the impressive 472 taxa described as new to science by Terry Erwin.

\section{Additions to the List of publications of Terry Erwin published after 2015}

For the list of publications before 2015 see ZooKeys Editorial Office (2015), https:// doi.org/10.3897/zookeys.541.7316.

Erwin TL, Kavanaugh DH, Maddison DR (2020) After 157 years, a second specimen and species of the phylogenetically enigmatic and previously monobasic genus Nototylus Gemminger \& Harold, 1868 (Coleoptera, Carabidae, Nototylini). ZooKeys 927: 65-74. https://doi.org/10.3897/zookeys.927.49584

Maddison DR, Kanda K, Boyd OF, Faille A, Porch N, Erwin TL, Roig-Juñent S (2019) Phylogeny of the beetle supertribe Trechitae (Coleoptera: Carabidae): unexpected clades, isolated lineages, and morphological convergence. Molecular Phylogenetics and Evolution 132: 151-176. https://doi.org/10.1016/j.ympev.2018.11.006

Erwin TL, Aldebron C (2018) Neotropical Thoasia Liebke, 1939 and Straneotia Mateu, 1961 of the Cryptobatida group, subtribe Agrina: Taxonomic revisions with notes on their ways of life (Insecta, Coleoptera, Carabidae, Lebiini). ZooKeys 742: 57-90. https://doi. org/10.3897/zookeys.742.22900

Erwin TL, Henry SC (2017) Hyboptera Chaudoir, 1872 of the Cryptobatida group of subtribe Agrina: A taxonomic revision with notes on their ways of life (Insecta, Coleoptera, Carabidae, Lebiini). ZooKeys 714: 61-127. https://doi.org/10.3897/zookeys.714.15113

Erwin TL, Zamorano LS, Geraci CJ (2017) Amazonian rainforests and their richness of Coleoptera, a dominant life form in the Critical Zone of the Neotropics. In: Foottit R, Adler P (Eds) Insect Biodiversity: Science and Society, $2^{\text {nd }}$ edn. Blackwell Publishing, New Jersey. https://doi.org/10.1002/9781118945568.ch4

Boyd OF, Erwin TL (2016) Taxonomic review of New World Tachyina (Coleoptera, Carabidae): descriptions of new genera, subgenera, and species, with an updated key to the subtribe in the Americas. ZooKeys 626: 87-123. https://doi.org/10.3897/zookeys.626.10033

García-Robledo C, Kuprewicz PK, Staines CL, Erwin TL, Kress WJ (2016) Limited tolerance by insects to high temperatures across tropical elevation gradients and the implications of global warming for extinction. Proceedings of the National Academy of Sciences of the United States of America 113(3): 680-685. https://doi.org/10.1073/pnas.1507681113

Levine NM, Zhang K, Longo M, Baccini A, Phillips OL, Lewis SL, Alvarez, E, de Andrade ACS, Brienen R, Erwin T, Feldpausch TR, Mendoza ALM, Vargas PN, Prieto A, Espejo JES, Malhi Y, Moorcroft PR (2016) Ecosystem heterogeneity determines the resilience of the Amazon to climate change. Proceedings of the National Academy of Sciences of the United States of America 113(3): 793-797. https://doi.org/10.1073/pnas.1511344112

Ashworth AC, Erwin TL (2016) Antarctotrechus balli sp. n. (Carabidae, Trechini): the first ground beetle from Antarctica. ZooKeys 635: 109-122. https://doi.org/10.3897/zookeys.635.10535 
Erwin TL, Micheli C, Chaboo CS (2015) Beetles (Coleoptera) of Peru: A Survey of the Families. Carabidae. Journal of the Kansas Entomological Society 88(2): 151-162. https://doi. org/10.2317/kent-88-02-151-162.1

Table I. Additions to the List of taxa named after Terry Erwin, published after 2015. See table 1 in: ZooKeys Editorial Office (2015), https://doi.org/10.3897/zookeys.541.7316.

\begin{tabular}{|c|c|c|c|c|c|c|c|}
\hline Order & Family & Genus & Species & Taxon author & $\begin{array}{c}\text { Publication } \\
\text { date }\end{array}$ & Reference & Locality \\
\hline Coleoptera & Carabidae & Erwinanillus & & $\begin{array}{c}\text { Giachino, } \\
\text { Eberhard \& Perina }\end{array}$ & $2021: 275$ & Giachino et al. (2021) & $\begin{array}{c}\text { WA, Southern } \\
\text { Goldfields region }\end{array}$ \\
\hline Coleoptera & Carabidae & Paratachys & terryli & Liebherr & 2021: 235 & Liebherr (2021) & USA: Hawaii \\
\hline Coleoptera & Carabidae & Diplocheila & erwini & $\begin{array}{l}\text { Allegro \& } \\
\text { Giachino }\end{array}$ & 2021: 438 & $\begin{array}{l}\text { Allegro and Giachino } \\
\qquad(2021)\end{array}$ & Cambodia \\
\hline Coleoptera & Carabidae & $\begin{array}{l}\text { subgenus } \\
\text { Erwinebria }\end{array}$ & & Kavanaugh & 2021: 120 & $\begin{array}{l}\text { Kavanaugh et al. } \\
\qquad(2021)\end{array}$ & North America \\
\hline Coleoptera & Curculionidae & Coptoborus & erwini & Smith \& Cognato & 2021: 651 & $\begin{array}{l}\text { Smith and Cognato } \\
\qquad(2021)\end{array}$ & Ecuador (Orellana) \\
\hline Coleoptera & Carabidae & Tasmanitachoides & erwini & $\begin{array}{l}\text { Maddison \& } \\
\text { Porch }\end{array}$ & 2021: 186 & $\begin{array}{l}\text { Maddison and Porch } \\
\qquad(2021)\end{array}$ & Australia: Tasmania \\
\hline Coleoptera & Carabidae & Yalongaphaenops & erwini & Belousov \& Kabak & 2021: 207 & $\begin{array}{l}\text { Belousov and Kabak } \\
\qquad(2021)\end{array}$ & $\begin{array}{c}\text { China, Sichuan } \\
\text { Province, }\end{array}$ \\
\hline Coleoptera & Chrysomelidae & Hemilactica & erwini & Konstantinov & 2021: 591 & Konstantinov (2021) & Dominican Republic \\
\hline Coleoptera & Curculionidae & Conotrachelus & terryerwini & Anderson & 2021: 723 & Anderson (2021) & Costa Rica \\
\hline Coleoptera & Carabidae & $\begin{array}{c}\text { Bembidion } \\
\text { (Ocydromus) }\end{array}$ & terryerwini & Neri \& Toledano & 2021: 222 & $\begin{array}{c}\text { Neri and Toledano } \\
(2021)\end{array}$ & Iran \\
\hline Coleoptera & Carabidae & Coptocarpus & erwini & $\begin{array}{c}\text { Will \& } \\
\text { Guéorguiev }\end{array}$ & 2021: 388 & $\begin{array}{l}\text { Will and Guéorguiev } \\
\qquad(2021)\end{array}$ & New Caledonia \\
\hline Coleoptera & Carabidae & Calleida & erwini & Casale & 2021: 497 & Casale (2021) & Peru, Madre de Dios \\
\hline Hymenoptera & Braconidae & Aleiodes & terryerwini & Sharkey & 2021: 582 & Sharkey et al. (2021) & Costa Rica \\
\hline Coleoptera & Histeridae & Phelister & erwini & $\begin{array}{l}\text { Caterino \& } \\
\text { Tishechkin }\end{array}$ & 2020: 25 & $\begin{array}{c}\text { Caterino and } \\
\text { Tishechkin (2020) }\end{array}$ & $\begin{array}{c}\text { Ecuador, Brasil, } \\
\text { Perú, Colombia, } \\
\text { Panama }\end{array}$ \\
\hline Hemiptera & Membracidae & Cladonota & erwini & Flynn & 2019: 413 & Flynn (2019) & Ecuador \\
\hline Hymenoptera & Braconidae & Zelomoprpha & terryerwini & Meierotto & 2019: 134 & Meierotto et al. (2019) & Costa Rica \\
\hline
\end{tabular}

Table 2. Additions to the List of genera and subgenera described by Terry Erwin after 2015. See table 3 in: ZooKeys Editorial Office (2015), https://doi.org/10.3897/zookeys.541.7316).

\begin{tabular}{|c|c|c|c|c|c|}
\hline Genus/Subgenus & Type species & Genus author & $\begin{array}{c}\text { Publication } \\
\text { date }\end{array}$ & Reference & Locality \\
\hline Antarctotrechus & $\begin{array}{l}\text { Antarctotrechus balli } \\
\text { Ashworth \& Erwin }\end{array}$ & $\begin{array}{l}\text { Ashworth \& } \\
\text { Erwin }\end{array}$ & 2016: 114 & Ashworth and Erwin (2016) & Antarctica, Oliver Bluffs \\
\hline Tachyxysta & $\begin{array}{l}\text { Tachyxysta howdenorum } \\
\text { Boyd \& Erwin }\end{array}$ & Boyd \& Erwin & 2016: 97 & Boyd and Erwin (2016) & Mexico, Honduras \\
\hline Stigmatachys & $\begin{array}{l}\text { Stigmatachys uvea Boyd } \\
\text { \& Erwin }\end{array}$ & Boyd \& Erwin & 2016: 111 & Boyd and Erwin (2016) & Perú \\
\hline Nothoderis & $\begin{array}{c}\text { Tachys rufotestacea } \\
\text { Hayward }\end{array}$ & Boyd \& Erwin & 2016: 113 & Boyd and Erwin (2016) & $\begin{array}{c}\text { North, Central and } \\
\text { Amazonian South America }\end{array}$ \\
\hline $\begin{array}{l}\text { Scolistichus (subgenus of } \\
\text { Meotachys) }\end{array}$ & $\begin{array}{c}\text { Meotachys riparius Boyd } \\
\text { \& Erwin }\end{array}$ & Boyd \& Erwin & 2016: 114 & Boyd and Erwin (2016) & $\begin{array}{l}\text { Amazon basin (Ecuador, } \\
\text { Brazil, Perú, Colombia) }\end{array}$ \\
\hline $\begin{array}{l}\text { Hylotachys (subgenus of } \\
\text { Meotachys) }\end{array}$ & $\begin{array}{l}\text { Meotachys ballorum Boyd } \\
\text { \& Erwin }\end{array}$ & Boyd \& Erwin & 2016: 115 & Boyd and Erwin (2016) & $\begin{array}{c}\text { Amazon basin (Ecuador, } \\
\text { Brazil, Perú) }\end{array}$ \\
\hline $\begin{array}{l}\text { Ammotachys (subgenus of } \\
\text { Elaphropus) }\end{array}$ & $\begin{array}{c}\text { Elaphropus marchantarius } \\
\text { Boyd \& Erwin }\end{array}$ & Boyd \& Erwin & 2016: 102 & Boyd and Erwin (2016) & $\begin{array}{c}\text { Amazon basin (Colombia, } \\
\text { Brasil, Venezuela) }\end{array}$ \\
\hline $\begin{array}{l}\text { Idiotachys (subgenus of } \\
\text { Elaphropus) }\end{array}$ & $\begin{array}{c}\text { Elaphropus acutifrons } \\
\text { Boyd \& Erwin }\end{array}$ & Boyd \& Erwin & 2016: 104 & Boyd and Erwin (2016) & Brazil \\
\hline
\end{tabular}


Table 3. Additions to the List of species described by Terry Erwin after 2015. See table 4 in: ZooKeys Editorial Office (2015), https://doi.org/10.3897/zookeys.541.7316.

\begin{tabular}{|c|c|c|c|c|c|}
\hline Genus & Species & Author & $\begin{array}{c}\text { Publication } \\
\text { date }\end{array}$ & Reference & Locality \\
\hline Nototylus & balli & Erwin \& Kavanaugh & 2020: 69 & $\begin{array}{l}\text { Erwin, Kavanaugh and } \\
\text { Maddison (2020) }\end{array}$ & French Guiana \\
\hline Thoasia & surinamensis & Erwin \& Aldebron & 2018: 68 & Erwin and Aldebron (2018) & Suriname \\
\hline Thoasia & pterosmaragdos & Aldebron \& Erwin & 2018: 69 & Erwin and Aldebron (2018) & French Guiana \\
\hline Thoasia & manu & Erwin \& Aldebron & 2018: 70 & Erwin and Aldebron (2018) & Ecuador, Perú \\
\hline Straneotia & confundis & Aldebron \& Erwin & 2018: 79 & Erwin and Aldebron (2018) & Ecuador \\
\hline Straneotia & moi & Aldebron \& Erwin & 2018: 83 & Erwin and Aldebron (2018) & French Guiana \\
\hline Straneotia & cylindroceps & Erwin \& Aldebron & 2018: 85 & Erwin and Aldebron (2018) & French Guiana \\
\hline Hyboptera & biolat & Erwin \& Henry & 2017: 82 & Erwin and Henry (2017) & Perú \\
\hline Hyboptera & vestiverdis & Henry \& Erwin & 2017: 83 & Erwin and Henry (2017) & Perú \\
\hline Hyboptera & scheelea & Erwin \& Henry & 2017: 87 & Erwin and Henry (2017) & Perú \\
\hline Hyboptera & shasta & Erwin & 2017: 88 & Erwin and Henry (2017) & Brazil \\
\hline Hyboptera & tiputini & Erwin \& Henry & 2017: 91 & Erwin and Henry (2017) & Ecuador \\
\hline Hyboptera & tepui & Erwin \& Henry & 2017: 92 & Erwin and Henry (2017) & Venezuela \\
\hline Hyboptera & lucida & Henry \& Erwin & 2017: 108 & Erwin and Henry (2017) & French Guiana \\
\hline Antarctotrechus & balli & Ashworth \& Erwin & 2016: 115 & Ashworth and Erwin (2016) & Antarctica, Oliver Bluffs \\
\hline Tachyxysta & howdenorum & Boyd \& Erwin & 2016: 98 & Boyd and Erwin (2016) & Mexico, Honduras \\
\hline Stigmatachys & uvea & Boyd \& Erwin & 2016: 112 & Boyd and Erwin (2016) & Perú \\
\hline Meotachys (Scolistichus) & riparius & Boyd \& Erwin & 2016: 115 & Boyd and Erwin (2016) & Colombia \\
\hline Meotachys (Hylotachys) & ballorum & Boyd \& Erwin & 2016: 116 & Boyd and Erwin (2016) & (Ecuador, Brazil, Colombia) \\
\hline Meotachys (Hylotachys) & rubrum & Boyd \& Erwin & 2016: 117 & Boyd and Erwin (2016) & Perú \\
\hline Elaphropus (Ammotachys) & marchantarius & Boyd \& Erwin & 2016: 103 & Boyd and Erwin (2016) & $\begin{array}{c}\text { Amazon basin (Colombia, } \\
\text { Brazil, Venezuela) }\end{array}$ \\
\hline Elaphropus (Idiotachys) & acutifrons & Boyd \& Erwin & 2016: 106 & Boyd and Erwin (2016) & Brazil \\
\hline Elaphropus (Nototachts) & occidentalis & Boyd \& Erwin & 2016: 107 & Boyd and Erwin (2016) & Perú, Brazil, Argentina \\
\hline
\end{tabular}

\section{References}

Allegro G, Giachino PM (2021) The genus Diplocheila Brullé, 1834 in Cambodia, with descriptions of two new species (Coleoptera, Carabidae, Licinini). In: Spence J, Casale A, Assmann T, Liebherr JK, Penev L (Eds) Systematic Zoology and Biodiversity Science: A tribute to Terry Erwin (1940-2020). ZooKeys 1044: 427-448. https://doi.org/10.3897/ zookeys. 1044.60072

Anderson RS (2021) Conotrachelus terryerwini, a majestic new species of Curculionidae (Molytinae, Conotrachelini) from Costa Rica. In: Spence J, Casale A, Assmann T, Liebherr JK, Penev L (Eds) Systematic Zoology and Biodiversity Science: A tribute to Terry Erwin (1940-2020). ZooKeys 1044: 721-727. https://doi.org/10.3897/zookeys.1044.62722

Ashworth AC, Erwin TL (2016) Antarctotrechus balli sp. n. (Carabidae, Trechini): the first ground beetle from Antarctica. ZooKeys 635: 109-122. https://doi.org/10.3897/zookeys.635.10535

Belousov IA, Kabak II (2021) Yalongaphaenops erwini gen. et sp. nov., the world's most highaltitude hypogean trechine beetle from China (Coleoptera, Carabidae, Trechinae). In: Spence J, Casale A, Assmann T, Liebherr JK, Penev L (Eds) Systematic Zoology and Biodiversity Science: A tribute to Terry Erwin (1940-2020). ZooKeys 1044: 197-220. https:// doi.org/10.3897/zookeys.1044.62572

Boyd OF, Erwin TL (2016) Taxonomic review of New World Tachyina (Coleoptera, Carabidae): descriptions of new genera, subgenera, and species, with an updated key to the subtribe in the Americas. ZooKeys 626: 87-123. https://doi.org/10.3897/zookeys.626.10033 
Casale A (2021) Biodiversity in tropical rainforests: Calleida Dejean 1825 at the BIOLAT Biological Station, Rio Manu, Peru, with descriptions of seven new species (Coleoptera Carabidae, Lebiini). Part 1. In: Spence J, Casale A, Assmann T, Liebherr JK, Penev L (Eds) Systematic Zoology and Biodiversity Science: A tribute to Terry Erwin (1940-2020). ZooKeys 1044: 479-510. https://doi.org/10.3897/zookeys.1044.64082

Caterino MS, Tishechkin AK (2020) Recognition and revision of the Phelister blairi group (Histeridae, Histerinae, Exosternini). ZooKeys 1001: 1-154. https://doi.org/10.3897/ zookeys. 1001.58447

Erwin TL, Aldebron C (2018) Neotropical Thoasia Liebke, 1939 and Straneotia Mateu, 1961 of the Cryptobatida group, subtribe Agrina: Taxonomic revisions with notes on their ways of life (Insecta, Coleoptera, Carabidae, Lebiini). ZooKeys 742: 57-90. https://doi. org/10.3897/zookeys.742.22900

Erwin TL, Henry SC (2017) Hyboptera Chaudoir, 1872 of the Cryptobatida group of subtribe Agrina: A taxonomic revision with notes on their ways of life (Insecta, Coleoptera, Carabidae, Lebiini). ZooKeys 714: 61-127. https://doi.org/10.3897/zookeys.714.15113

Erwin TL, Kavanaugh DH, Maddison DR (2020) After 157 years, a second specimen and species of the phylogenetically enigmatic and previously monobasic genus Nototylus Gemminger \& Harold, 1868 (Coleoptera, Carabidae, Nototylini). ZooKeys 927: 65-74. https://doi.org/10.3897/zookeys.927.49584

Erwin T, Stoev P, Penev L (2018) ZooKeys anniversary: 10 years of leadership toward openaccess publishing of zoological data and establishment at Pensoft of like-minded sister journals across the biodiversity spectrum. ZooKeys 770: 1-8. https://doi.org/10.3897/ zookeys.770.28105

Flynn D (2019) Review of the genus Cladonota Stål with keys, illustrations of adults, and description of a new species from Ecuador (Hemiptera: Membracidae: Membracinae: Hypsoprorini). II. Subgenus Cladonota Stål. Proceedings of the Entomological Society of Washington 121(3): 405-428. https://doi.org/10.4289/0013-8797.121.3.405

Giachino PM, Eberhard S, Perina G (2021) A rich fauna of subterranean short-range endemic Anillini (Coleoptera, Carabidae, Trechinae) from semi-arid regions of Western Australia. In: Spence J, Casale A, Assmann T, Liebherr JK, Penev L (Eds) Systematic Zoology and Biodiversity Science: A tribute to Terry Erwin (1940-2020). ZooKeys 1044: 269-337. https://doi.org/10.3897/zookeys.1044.58844

Kavanaugh DH, Maddison DR, Simison WB, Schoville SD, Schmidt J, Faille A, Moore W, Pflug JM, Archambeault SL, Hoang T, Chen J-Y (2021) Phylogeny of the supertribe Nebriitae (Coleoptera, Carabidae) based on analyses of DNA sequence data. In: Spence J, Casale A, Assmann T, Liebherr JK, Penev L (Eds) Systematic Zoology and Biodiversity Science: A tribute to Terry Erwin (1940-2020). ZooKeys 1044: 41-152. https://doi. org/10.3897/zookeys.1044.62245

Konstantinov AS (2021) Flea beetles of the West Indies: the genus Hemilactica Blake, 1937 (Coleoptera, Chrysomelidae, Galerucinae, Alticini). In: Spence J, Casale A, Assmann T, Liebherr JK, Penev L (Eds) Systematic Zoology and Biodiversity Science: A tribute to Terry Erwin (1940-2020). ZooKeys 1044: 589-607. https://doi.org/10.3897/zookeys.1044.62632

Liebherr JK (2021) Hawaiian Paratachys Casey (Coleoptera, Carabidae): small beetles of sodden summits, stony streams, and stygian voids. In: Spence J, Casale A, Assmann T, Liebherr 
JK, Penev L (Eds) Systematic Zoology and Biodiversity Science: A tribute to Terry Erwin (1940-2020). ZooKeys 1044: 229-268. https://doi.org/10.3897/zookeys.1044.59674

Maddison DR, Porch N (2021) A preliminary phylogeny and review of the genus Tasmanitachoides, with descriptions of two new species (Coleoptera, Carabidae, Bembidarenini). In: Spence J, Casale A, Assmann T, Liebherr JK, Penev L (Eds) Systematic Zoology and Biodiversity Science: A tribute to Terry Erwin (1940-2020). ZooKeys 1044: 151-194. https:// doi.org/10.3897/zookeys.1044.62253

Meierotto S, Sharkey MJ, Janzen DH, Hallwachs W, Hebert PDN, Chapman EG, Smith MA (2019) A revolutionary protocol to describe understudied hyperdiverse taxa and overcome the taxonomic impediment. Deutsche Entomologische Zeitschrift 66(2): 119-145. https://doi.org/10.3897/dez.66.34683

Neri P, Toledano L (2021) Bembidion (Ocydromus) terryerwini sp. nov. from Iran (Coleoptera, Carabidae, Bembidiina). In: Spence J, Casale A, Assmann T, Liebherr JK, Penev L (Eds) Systematic Zoology and Biodiversity Science: A tribute to Terry Erwin (1940-2020). ZooKeys 1044: 221-228. https://doi.org/10.3897/zookeys.1044.63607

Sharkey MJ, Janzen DH, Hallwachs W, Chapman EG, Smith MA, Dapkey T, Brown A, Ratnasingham S, Naik S, Manjunath R, Perez K, Milton M, Hebert P, Shaw SR, Kittel RN, Solis MA, Metz MA, Goldstein PZ, Brown JW, Quicke DLJ, van Achterberg C, Brown BV, Burns JM (2021) Minimalist revision and description of 403 new species in 11 subfamilies of Costa Rican braconid parasitoid wasps, including host records for 219 species. ZooKeys 1013: 1-665. https://doi.org/10.3897/zookeys.1013.55600

Smith SM, Cognato AI (2021) A Revision of the Neotropical genus Coptoborus Hopkins (Coleoptera, Curculionidae, Scolytinae, Xyleborini). In: Spence J, Casale A, Assmann T, Liebherr JK, Penev L (Eds) Systematic Zoology and Biodiversity Science: A tribute to Terry Erwin (1940-2020). ZooKeys 1044: 609-720. https://doi.org/10.3897/zookeys. 1044.62246

Will K, Guéorguiev B (2021) Phylogenetic systematics of the genera of Thryptocerina Jeannel, 1949 and new species from New Caledonia (Coleoptera, Carabidae, Oodini). In: Spence J, Casale A, Assmann T, Liebherr JK, Penev L (Eds) Systematic Zoology and Biodiversity Science: A tribute to Terry Erwin (1940-2020). ZooKeys 1044: 375-425. https://doi. org/10.3897/zookeys.1044.63775 